\title{
In-hospital mortality associated with community-acquired pneumonia due to methicillin-resistant Staphylococcus aureus: a matched-pair cohort study
}

Yukiyo Sakamoto ${ }^{1}$, Yasuhiro Yamauchi ${ }^{1}$, Taisuke Jo ${ }^{1,2^{*}}$, Nobuaki Michihata ${ }^{2}$, Wakae Hasegawa ${ }^{1}$, Hideyuki Takeshima ${ }^{1,3}$, Hiroki Matsui ${ }^{4}$, Kiyohide Fushimi ${ }^{5}$, Hideo Yasunaga ${ }^{4}$ and Takahide Nagase ${ }^{1}$

\begin{abstract}
Background: It remains unclear whether methicillin-resistant Staphylococcus aureus (MRSA) pneumonia is associated with higher mortality compared with non-MRSA pneumonia. This study's objective was to compare outcomes including in-hospital mortality and healthcare costs during hospitalisation between patients with MRSA pneumonia and those with non-MRSA pneumonia.

Methods: Using a national inpatient database in Japan, we conducted a 1:4 matched-pair cohort study of inpatients with community-acquired pneumonia from 1 April 2012 to 31 March 2014. In-hospital outcomes (mortality, length of stay and healthcare costs during hospitalisation) were compared between patients with and without MRSA infection. We performed multiple imputation using chained equations followed by multivariable regression analyses fitted with generalised estimating equations to account for clustering within matched pairs. All-cause in-hospital mortality and healthcare costs during hospitalisation were compared for pneumonia patients with and without MRSA infection.

Results: Of 450,317 inpatients with community-acquired pneumonia, 3102 patients with MRSA pneumonia were matched with 12,320 patients with non-MRSA pneumonia. The MRSA pneumonia patients had higher mortality, longer hospital stays and higher costs. Multivariable logistic regression analysis revealed that MRSA pneumonia was significantly associated with higher in-hospital mortality compared with non-MRSA pneumonia (adjusted odds ratio $=1.94 ; 95 \%$ confidence interval: $1.72-2.18 ; p<0.001$ ). Healthcare costs during hospitalisation were significantly higher for patients with MRSA pneumonia than for those with non-MRSA pneumonia (difference = USD 8502; 95\% confidence interval: USD 7959-9045; $p<0.001)$.
\end{abstract}

Conclusions: MRSA infection was associated with higher in-hospital mortality and higher healthcare costs during hospitalisation, suggesting that preventing MRSA pneumonia is essential.

Keywords: Methicillin-resistant Staphylococcus aureus pneumonia, Community-acquired pneumonia, In-hospital mortality, Healthcare costs during hospitalisation

*Correspondence: jo-taisuke@umin.ac.jp

1 Department of Respiratory Medicine, Graduate School of Medicine, The University of Tokyo, 7-3-1, Hongo, Bunkyo-ku, Tokyo 113-8655, Japan

Full list of author information is available at the end of the article

\section{Introduction}

Methicillin-resistant Staphylococcus aureus (MRSA) is a drug-resistant bacterium. The World Health Organization reported that MRSA infections accounted for 
$51.3 \%$ and 53\% of Staphylococcus aureus infections in the United States and Japan, respectively [1].

Mortality from pneumonia caused by multidrug-resistant pathogens has been reported to be higher than that from pneumonia caused by other pathogens [2]; however, it remains unclear whether mortality from MRSA pneumonia is higher than mortality from non-MRSA pneumonia. Only a few studies have compared mortality between MRSA pneumonia and non-MRSA pneumonia; one showed a trend toward higher in-hospital mortality among MRSA pneumonia patients [3], whereas another showed that MRSA infection did not affect intensive care unit (ICU) mortality or in-hospital mortality in patients with ventilator-associated pneumonia [4]. These previous studies did not adjust for pulmonary comorbidities, which have been reported to be associated with in-hospital mortality in hospitalised patients with pneumonia [5].

The aim of the present study was to use a national inpatient database to examine the differences in in-hospital mortality and healthcare costs during hospitalisation between patients with MRSA pneumonia and those with non-MRSA pneumonia, adjusting for pulmonary comorbidities.

\section{Methods}

\section{Data source}

We used the Diagnosis Procedure Combination database, a nationwide inpatient administrative claims and discharge abstract database in Japan. This database contains data on main diagnoses, primary diagnosis, and comorbidities at admission, recorded using International Classification of Diseases and Related Health Problems, 10th Revision (ICD-10) codes and Japanese text data; age; sex; body height and weight; grade of activities of daily life on admission (Barthel Index) [6]; level of dyspnoea based on the Hugh-Jones classification [7]; ambulance use and discharge status. The database also includes the Age, Dehydration, Respiratory Failure, Orientation Disturbance and Blood Pressure (A-DROP) score for patients with community-acquired pneumonia, as well as data on whether C-reactive protein (CRP) was $\geq 20 \mathrm{mg} / \mathrm{dL}$ or infiltration covering at least two-thirds of one lung on chest radiography, mechanical ventilation during hospitalisation and hospitalisation costs. The sensitivity and specificity of the recorded ICD-10 codes and procedures in the Diagnosis Procedure Combination database were validated in a previous study [8].

The Hugh-Jones classification is a widely used dyspnoea scale with the following categories: I (the patient's breathing is as good as that of other people of own age and build while working, walking and climbing hills or stairs), II (the patient is able to walk at the pace of normal people of the same age and build on level ground but is unable to keep up on hills or stairs), III (the patient is unable to keep up with normal people on level ground but is able to walk about a mile or more at their own pace), IV (the patient is unable to walk more than about 50 yards on level ground without resting), $\mathrm{V}$ (the patient is short of breath when talking or undressing or is unable to leave their home because of shortness of breath) and unspecified (the patient cannot be classified into any of the above grades because of bedridden status) [7].

The A-DROP score, established by the Japanese Respiratory Society, is a modified version of the CURB-65 (Confusion, Urea, Respiratory rate, Blood pressure-65) score [9]. The A-DROP score includes the following parameters: age (men: $\geq 70$ years, women: $\geq 75$ years), dehydration (blood urea nitrogen $\geq 21 \mathrm{mg} / \mathrm{dL}$ ), respiratory failure $\left(\mathrm{SaO}_{2} \leq 90 \%\right.$ or $\left.\mathrm{PaO}_{2} \leq 60 \mathrm{mmHg}\right)$, orientation disturbance (confusion) and low blood pressure (systolic blood pressure $\leq 90 \mathrm{mmHg}$ ).

The Institutional Review Board of The University of Tokyo approved this study and waived the requirement for patient informed consent because of the anonymous nature of the data.

\section{Patient selection}

We retrospectively collected data on patients admitted to hospitals for community-acquired pneumonia who were discharged from 1 April 2012 to 31 March 2014. Community-acquired pneumonia was defined according to the 2019 guidelines on the management of adults with community-acquired pneumonia published by the Infectious Diseases Society of America/American Thoracic Society [10].

We defined MRSA pneumonia patients as those who had both the ICD-10 code for MRSA pneumonia and records of the administration of anti-MRSA antibiotics (vancomycin, linezolid, teicoplanin or arbekacin) for more than 7 days.

\section{Outcomes}

The primary outcome of this study was all-cause in-hospital mortality. The secondary outcomes were 30-day inhospital mortality, 90-day in-hospital mortality, length of stay and hospitalisation costs. The duration of antibiotic therapy was also evaluated.

\section{Statistical analysis}

The $\chi^{2}$ test was used to compare proportions between groups. The two-sample $t$-test was used to compare average values, and the Mann-Whitney test was used to compare the median values between groups.

Among the patients with pneumonia, we selected an MRSA pneumonia group and a non-MRSA pneumonia group with 1:4 matching: for each patient in the MRSA 
pneumonia group, we identified four non-MRSA patients of the same sex who were admitted to the same hospital in the same year and whose ages were within 5 years of the age of the MRSA patient. We used hospital identifiers for matching to cancel out site-specific effects such as physician practice patterns and treatment outcomes [11].

We performed multiple imputation for missing data on body mass index (BMI), Barthel Index, Hugh-Jones grade, A-DROP score, CRP $\geq 20 \mathrm{mg} / \mathrm{mL}$ or infiltration covering at least two-thirds of one lung on chest radiography, and hospitalisation costs. We replaced each missing value with a set of substituted plausible values by generating 20 complete datasets using the multivariate imputation by chained equations method. The following covariates were used to create these 20 complete datasets: MRSA pneumonia, age, sex, fiscal year, haemodialysis, mechanical ventilation at admission, ICU admission, arrival by ambulance, chronic obstructive pulmonary disease (COPD), interstitial lung disease, aspiration pneumonia, Pseudomonas aeruginosa pneumonia, cerebrovascular disease, Parkinson disease, diabetes, dementia, in-hospital death, 30-day in-hospital death and 90-day in-hospital death, with the assumption that data were missing at random [12, 13]. Estimates from these 20 imputed datasets were combined using Rubin's rule to obtain combined imputation estimates and standard errors.

Then, using multivariable logistic regression analysis fitted with generalised estimating equations to account for the 1:4 matched-pair clustering, we examined the factors associated with all-cause in-hospital mortality. Multiple linear regression analysis fitted with generalised estimating equations was also used to assess hospitalisation costs. The following independent variables were included in the models: age, sex, MRSA pneumonia, BMI, Barthel Index, Hugh-Jones grade, A-DROP score, $\mathrm{CRP} \geq 20 \mathrm{mg} / \mathrm{mL}$ or infiltration covering at least twothirds of one lung on chest radiography, haemodialysis, mechanical ventilation at admission, ICU admission, arrival by ambulance, COPD, interstitial lung disease, aspiration pneumonia and Pseudomonas aeruginosa pneumonia. For sensitivity analyses, we added independent variables of chronic heart failure, chronic liver disease, sepsis, acute renal failure, leukopenia, immunosuppression and stroke to the multivariable regression models used in the main analyses. Statistical analyses were performed using SPSS, Version 22.0 (IBM SPSS, Armonk, NY, USA) and Stata, Version 16 (StataCorp, College Station, TX, USA).

\section{Patient and public involvement}

Patients or the public were not involved in the design, or conduct, or reporting, or dissemination plans of our research.

\section{Results}

A total of 450,317 patients were hospitalised for community-acquired pneumonia during the study period, including 3102 patients with MRSA pneumonia. There were 44,854 in-hospital deaths (10.0\%). The patients with MRSA pneumonia were matched with 12,320 patients hospitalised for non-MRSA pneumonia. The mean age was 79.4 years (standard deviation $[\mathrm{SD}]=10.8$ ), and $66.4 \%$ of the patients were male. Table 1 shows the characteristics of patients with MRSA pneumonia and non-MRSA pneumonia after 1:4 matching. The MRSA pneumonia group tended to have lower BMIs, lower activities of daily living scores, higher Hugh-Jones grades, higher A-DROP scores, CRP $\geq 20 \mathrm{mg} / \mathrm{mL}$ or infiltration covering at least two-thirds of one lung on chest radiography, mechanical ventilation on the day of admission or the following day, ICU admission on the day of admission or the following day, ambulance use, aspiration pneumonia and end-stage renal disease with haemodialysis. The percentages of patients with smoking history and COPD were lower in the MRSA pneumonia group than in the non-MRSA pneumonia group.

All-cause in-hospital mortality was $31.2 \%$ in the MRSA pneumonia group, whereas it was $11.6 \%$ in the nonMRSA pneumonia group (Table 2). All-cause 30-day and 90-day mortality were also higher in the MRSA pneumonia group than in the non-MRSA pneumonia group. The duration of antibiotic therapy was longer in the MRSA pneumonia group than in the non-MRSA pneumonia group. Length of hospital stay was longer and hospitalisation costs were higher in the MRSA pneumonia group than in the non-MRSA pneumonia group.

The percentage of patients with missing data on HughJones grade was $25.1 \%$ for all patients with pneumonia, and this number was higher for patients with MRSA pneumonia (37.5\%) before multiple imputation. Hospitalisation costs data were missing for $0.2 \%$ of all patients with pneumonia.

Table 3 shows the results of the multivariable logistic regression analysis with generalised estimating equations after multiple imputation for all-cause in-hospital mortality. MRSA pneumonia was significantly associated with higher mortality compared with non-MRSA pneumonia (adjusted odds ratio $=1.94 ; 95 \%$ confidence interval: $1.72-2.18 ; p<0.001)$.

Higher mortality was significantly associated with lower BMI, lower Barthel Index, higher Hugh-Jones grade, higher A-DROP score, CRP $\geq 20 \mathrm{mg} / \mathrm{dL}$ or infiltration of least two-thirds of one lung, mechanical ventilation at admission, interstitial lung disease and aspiration pneumonia. The sensitivity analyses showed similar results to the main analyses (Additional file 1: Tables S1 and S2). 
Table 1 Characteristics of patients with MRSA pneumonia and patients with non-MRSA pneumonia after 1:4 matching

\begin{tabular}{|c|c|c|c|c|c|}
\hline & \multicolumn{2}{|c|}{ MRSA pneumonia } & \multicolumn{2}{|c|}{ Non-MRSA pneumonia } & \multirow[t]{2}{*}{$p$-value } \\
\hline & $n(\%)$ & & $n(\%)$ & & \\
\hline Sex & & & & & 0.943 \\
\hline Male & 2059 & $(66.4)$ & 8186 & $(66.4)$ & \\
\hline Female & 1043 & $(33.6)$ & 4134 & (33.6) & \\
\hline Age (years, mean $\pm S D$ ) & $79.6 \pm 10.7$ & & $79.5 \pm 10.5$ & & 0.698 \\
\hline Body mass index $\left(\mathrm{kg} / \mathrm{m}^{2}\right)$ & & & & & $<0.001$ \\
\hline$<18.5$ & 1388 & $(44.7)$ & 3539 & $(28.7)$ & \\
\hline $18.5-24.9$ & 1136 & $(36.6)$ & 5899 & $(47.9)$ & \\
\hline $25-29.9$ & 116 & $(3.7)$ & 1147 & $(9.3)$ & \\
\hline$\geq 30$ & 28 & $(0.9)$ & 199 & $(1.6)$ & \\
\hline Missing & 434 & $(14.0)$ & 1536 & $(12.5)$ & \\
\hline ADL score (Barthel Index) & & & & & $<0.001$ \\
\hline $85-100$ & 474 & $(15.3)$ & 4144 & $(33.4)$ & \\
\hline $60-80$ & 159 & $(5.1)$ & 991 & $(8.0)$ & \\
\hline $0-55$ & 2024 & $(65.2)$ & 5503 & $(44.7)$ & \\
\hline Missing & 445 & $(14.3)$ & 1712 & $(13.9)$ & \\
\hline Smoking history & & & & & 0.003 \\
\hline Yes & 1226 & $(39.5)$ & 5239 & $(42.5)$ & \\
\hline No & 1876 & $(60.5)$ & 7086 & $(57.5)$ & \\
\hline Hugh-Jones grade & & & & & $<0.001$ \\
\hline । & 132 & $(4.3)$ & 1604 & $(13.0)$ & \\
\hline$\|$ & 193 & $(6.2)$ & 1774 & $(14.4)$ & \\
\hline III & 231 & $(7.5)$ & 1411 & $(11.5)$ & \\
\hline IV & 435 & $(14.0)$ & 2444 & $(19.8)$ & \\
\hline V & 948 & $(30.6)$ & 2375 & $(19.3)$ & \\
\hline Missing & 1163 & $(37.5)$ & 2712 & $(22.0)$ & \\
\hline A-DROP score & & & & & $<0.001$ \\
\hline 0 & 162 & $(5.2)$ & 1089 & $(8.8)$ & \\
\hline 1 & 639 & $(20.6)$ & 3882 & $(31.5)$ & \\
\hline 2 & 870 & $(28.0)$ & 3871 & $(31.4)$ & \\
\hline 3 & 701 & $(22.6)$ & 2099 & $(17.0)$ & \\
\hline 4 & 410 & $(13.2)$ & 791 & $(6.4)$ & \\
\hline 5 & 218 & $(7.0)$ & 316 & $(2.6)$ & \\
\hline Missing & 102 & (3.3) & 272 & $(2.2)$ & \\
\hline Dehydration & & & & & $<0.001$ \\
\hline Yes & 1788 & $(55.6)$ & 5443 & $(44.2)$ & \\
\hline No & 1288 & $(41.5)$ & 6779 & $(55.0)$ & \\
\hline Missing & 26 & $(0.8)$ & 98 & $(0.8)$ & \\
\hline Respiratory failure & & & & & $<0.001$ \\
\hline Yes & 1612 & $(52.0)$ & 4591 & $(37.3)$ & \\
\hline No & 1468 & $(47.3)$ & 7642 & $(62.0)$ & \\
\hline Missing & 22 & $(0.7)$ & 87 & $(0.7)$ & \\
\hline Orientation disturbance & & & & & $<0.001$ \\
\hline Yes & 941 & $(30.3)$ & 1934 & $(15.7)$ & \\
\hline No & 2100 & $(67.7)$ & 10,285 & $(83.5)$ & \\
\hline Missing & 61 & $(2.0)$ & 101 & $(0.8)$ & \\
\hline Systolic blood pressure & & & & & $<0.001$ \\
\hline$<90 \mathrm{mmHg}$ & 502 & $(16.2)$ & 1013 & $(8.2)$ & \\
\hline$\geq 90 \mathrm{mmHg}$ & 2581 & $(83.2)$ & 11,243 & $(91.3)$ & \\
\hline
\end{tabular}


Table 1 (continued)

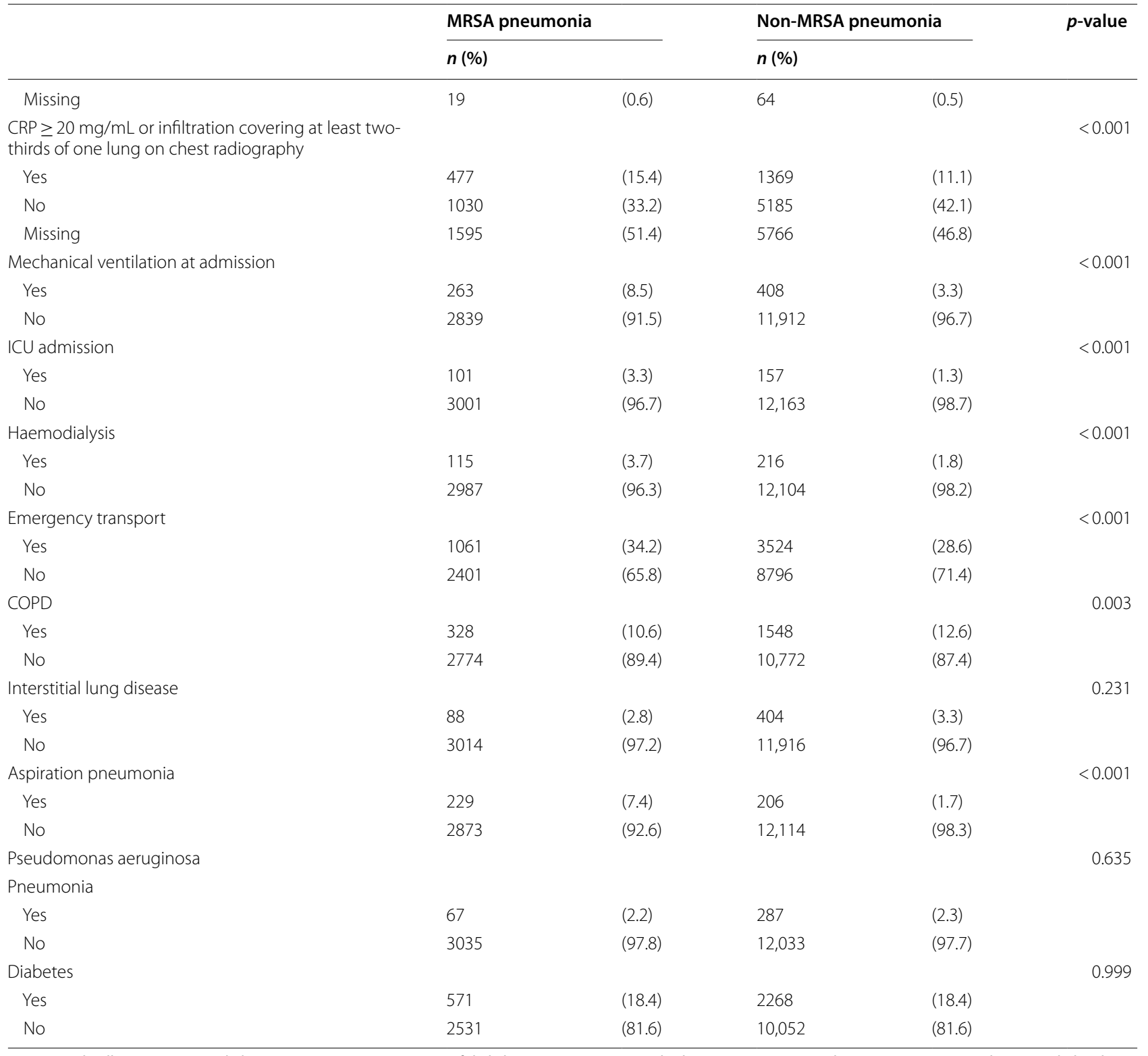

MRSA, methicillin-resistant Staphylococcus aureus; ADL, activities of daily living; A-DROP, Age, Dehydration, Respiratory Failure, Orientation Disturbance and Blood Pressure; CRP, C-reactive protein; ICU, intensive care unit; COPD; chronic obstructive pulmonary disease

Table 2 Outcomes of patients with MRSA pneumonia and patients with non-MRSA pneumonia after 1:4 matching

\begin{tabular}{|c|c|c|c|c|c|}
\hline \multirow[b]{2}{*}{ In-hospital mortality } & \multicolumn{2}{|c|}{ MRSA pneumonia } & \multicolumn{2}{|c|}{ Non-MRSA pneumonia } & \multirow{2}{*}{$\frac{p \text {-value }}{<0.001}$} \\
\hline & 967 & $(31.2)$ & 1429 & $(11.6)$ & \\
\hline 30-day mortality & 382 & $(12.3)$ & 1011 & $(8.20)$ & $<0.001$ \\
\hline 90-day mortality & 809 & $(26.1)$ & 1330 & $(10.8)$ & $<0.001$ \\
\hline Length of stay (days) & 35.0 & {$[22-62]$} & 14.0 & [9-23] & $<0.001$ \\
\hline Antibiotic therapy (days) & 24.0 & {$[15-45]$} & 9.0 & {$[6-13]$} & $<0.001$ \\
\hline Hospitalisation costs (USD) & 12,156 & {$[7827-20,615]$} & 4665 & [3163-7298] & $<0.001$ \\
\hline
\end{tabular}

Data are expressed as numbers (\%) or as medians [interquartile ranges]

MRSA, methicillin-resistant Staphylococcus aureus; USD, United States Dollar 
Table 3 Multivariable logistic regression analysis with generalised estimating equations accounting for clustering within matched pairs for all-cause in-hospital mortality

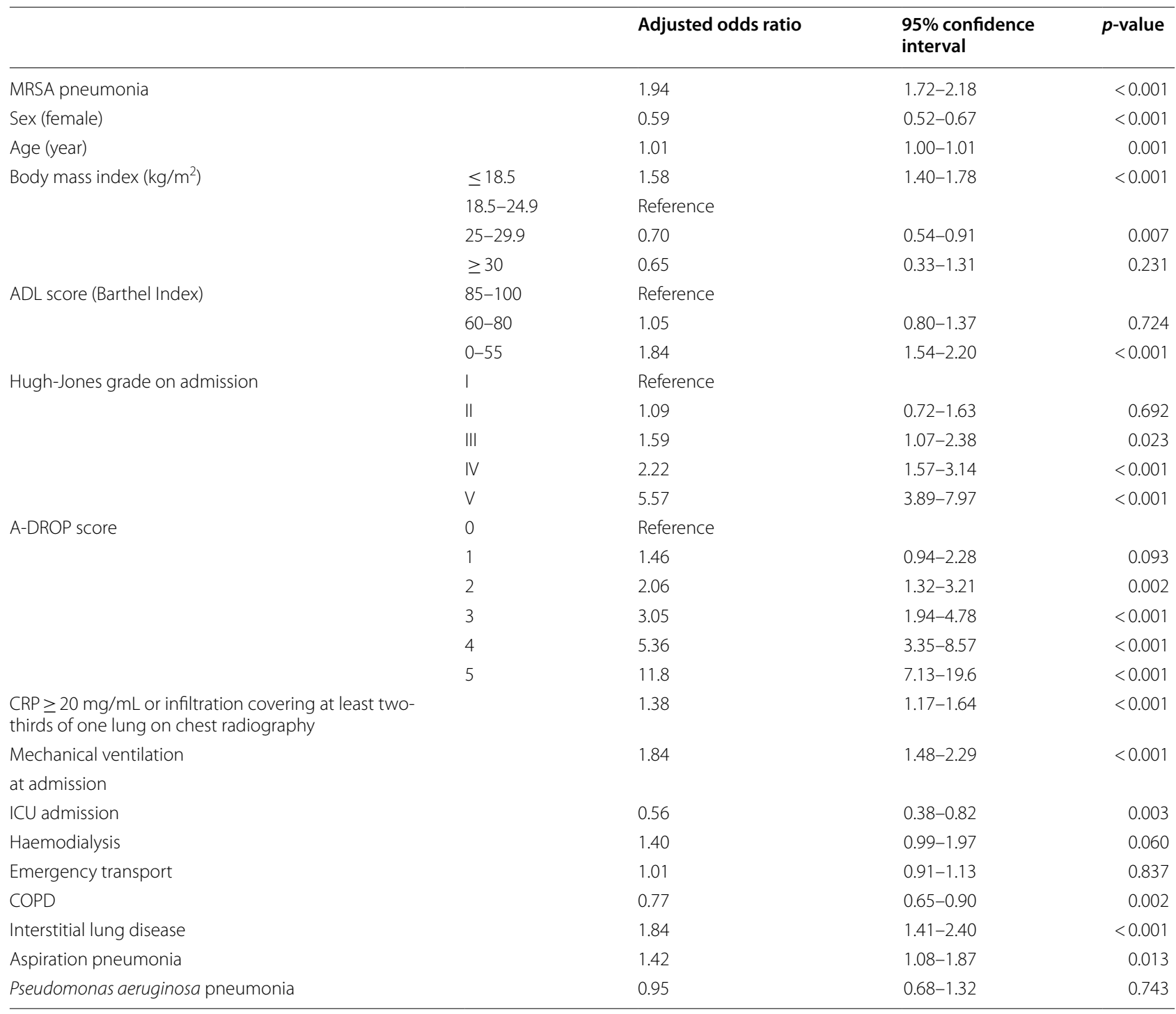

MRSA, methicillin-resistant Staphylococcus aureus; ADL, activities of daily living; A-DROP, Age, Dehydration, Respiratory Failure, Orientation Disturbance and Blood Pressure; CRP, C-reactive protein; ICU, intensive care unit; COPD, chronic obstructive pulmonary disease

We performed the multiple linear regression analysis with generalised estimating equations after multiple imputation for hospitalisation costs. Hospitalisation costs were significantly higher for patients with MRSA pneumonia than for those with non-MRSA pneumonia (difference $=$ United States Dollar (USD) 8502; 95\% confidence interval: USD 7959-9045; $p<0.001$ ). In the sensitivity analyses, hospitalisation costs were significantly higher for patients with MRSA pneumonia than for those with non-MRSA pneumonia (difference $=$ USD 8457; 95\% confidence interval: USD 7919-8996; $p<0.001)$.

\section{Discussion}

Using a nationwide inpatient database in Japan, our study showed that mortality was higher in patients with MRSA pneumonia than in those with non-MRSA pneumonia. In addition, we showed that hospitalisation costs were higher for patients with MRSA pneumonia than for those with non-MRSA pneumonia.

In our study, in-hospital mortality among patients with MRSA pneumonia was $31.2 \%$. Previous studies have reported MRSA pneumonia mortality to be around $30 \%[14,15]$, which is comparable to our results. 
Studies have shown conflicting results on the difference in mortality between patients with MRSA pneumonia and those with non-MRSA pneumonia. Some studies have shown higher mortality for patients with pneumonia caused by multidrug-resistant pathogens than for those with other types of pneumonia [2, 14], whereas other studies have found no significant differences [4, 16]. Several studies have shown high mortality in patients with MRSA bacteraemia [17-19], but few studies have focused on MRSA pneumonia. In the present study, we clearly demonstrated that mortality was twice as high in patients with MRSA pneumonia than in patients with non-MRSA pneumonia.

Additionally, previous studies have shown conflicting results on the difference in hospitalisation costs between MRSA and methicillin-sensitive Staphylococcus aureus pneumonia [20-22]. We confirmed that healthcare costs were higher for MRSA pneumonia than for non-MRSA pneumonia, including methicillin-sensitive Staphylococcus aureus pneumonia. Longer hospital stay may lead to higher hospitalisation costs in patients with MRSA pneumonia. Possible causes of the longer hospital stay are that patients with MRSA pneumonia are frailer and require longer duration of antibiotic therapy. The patients with MRSA pneumonia tended to have lower BMIs and lower activities of daily living scores. Although we adjusted for BMI and activities of daily living score, we were unable to fully evaluate frailty because the database lacked data on other components of the frailty definition, such as grip strength, exhaustion and slowness of walking [23].

Previous studies have shown several pulmonary comorbidities to be associated with higher mortality in patients with pneumonia, including interstitial lung disease $[5,24]$ and aspiration pneumonia [25]. The association between COPD and mortality remains uncertain in hospitalised adult patients with pneumonia $[5,26]$. Our multivariable regression analysis included these comorbidities, finding no significant association between COPD and in-hospital mortality.

Limitations should be acknowledged. First, the database used for this study does not include bacterial culture or drug-susceptibility test results. We therefore combined an MRSA diagnosis and treatment for MRSA to identify patients with MRSA pneumonia. Second, the database does not include pulmonary function test results; thus, we were not able to account for the severity of pulmonary comorbidities. Finally, several factors, such as previous antibiotic use, were unmeasured, and we therefore could not eliminate confounding biases arising from these factors.

In conclusion, adjusted in-hospital mortality and hospitalisation costs were significantly higher for patients with MRSA pneumonia than for those with non-MRSA pneumonia in this matched-pair cohort study.

\section{Abbreviations}

MRSA: Methicillin-resistant Staphylococcus aureus; ICU: Intensive care unit; ICD10: The International Classification of Disease and Related Health Problems, 10th Revision; A-DROP: Age-Dehydration-Respiratory failure-Orientation disturbance-blood Pressure; CRP: C-reactive protein; CURB-65: ConfusionUrea-Respiratory rate-Blood pressure-65; BMI: Body mass index; COPD: Chronic obstructive pulmonary disease; SD: Standard deviation; USD: United States Dollar.

\section{Supplementary Information}

The online version contains supplementary material available at https://doi. org/10.1186/s12890-021-01713-1.

Additional file 1. Table S1. Characteristics of patients with MRSA pneumonia and patients with non-MRSA pneumonia after 1:4 matching regarding with chronic heart failure, chronic liver diseases, sepsis, acute renal failure, leukopenia,immunosupression and stroke. Table S2; Sensitivity analyses adjusted comorbidities including chronic heart failure, chronicliver diseases, sepsis, acute renal failure, leukopenia,immunosupression and stroke for all-cause in-hospital mortality.

\section{Acknowledgements}

Not applicable.

\section{Authors' contributions}

TJ had full access to all of the data in the study and takes responsibility for the integrity of the data and the accuracy of the data analysis, including and especially any adverse effects. YS contributed substantially to the study design, data analysis and interpretation, and the writing of the manuscript. HY contributed substantially to the data analysis and interpretation, and the writing of the manuscript. NM, WH, HT, HM, KF, YY and TN contributed substantially to the study design, data analysis and interpretation. All authors read and approved the final manuscript.

\section{Funding}

This work was supported by grants from the Ministry of Health, Labour and Welfare, Japan (19AA2007 and 20AA2005) and the Ministry of Education, Culture, Sports, Science and Technology, Japan (20H03907). The funding bodies had no role in the design of the study; the collection, analysis or interpretation of the data; or the writing of the manuscript.

\section{Availability of data and materials}

The datasets generated and/or analysed during the current study are not publicly available.

\section{Declarations}

Ethics approval and consent to participate

Not applicable.

\section{Consent for publication}

Not applicable.

\section{Competing interests}

The authors declare that they have no competing interests.

\section{Author details}

${ }^{1}$ Department of Respiratory Medicine, Graduate School of Medicine, The University of Tokyo, 7-3-1, Hongo, Bunkyo-ku, Tokyo 113-8655, Japan. ²Department of Health Services Research, Graduate School of Medicine, The University of Tokyo, 7-3-1, Hongo, Bunkyo-ku, Tokyo 113-8655, Japan. ${ }^{3}$ Department 
of Respiratory Medicine, NTT Medical Center Tokyo, 5-9-22, Higashi-Gotanda Shinagawa-ku, Tokyo 141-8625, Japan. ${ }^{4}$ Department of Clinical Epidemiology and Health Economics, School of Public Health, The University of Tokyo, 7-3-1, Hongo, Bunkyo-ku, Tokyo 113-8655, Japan. ${ }^{5}$ Department of Health Policy and Informatics, Tokyo Medical and Dental University Graduate School of Medicine, Tokyo, 1-5-45 Yushima, Bunkyo-ku, Tokyo 113-8510, Japan.

Received: 15 June 2021 Accepted: 26 October 2021

Published online: 03 November 2021

\section{References}

1. World Health Organization. Antimicrobial Resistance: Global Report on Surveillance. 2014. https://apps.who.int/iris/bitstream/10665/112642/1/ 9789241564748_eng.pdf?ua=1. Accessed 7 Feb 2020.

2. Falcone M, Russo A, Giannella M, Cangemi R, Scarpellini MG, Bertazzoni G, et al. Individualizing risk of multidrug-resistant pathogens in communityonset pneumonia. PLOS ONE. 2015;10:e0119528.

3. Jacobs DM, Shaver A. Prevalence of and outcomes from Staphylococcus aureus pneumonia among hospitalized patients in the United States, 2009-2012. Am J Infect Control. 2017;45:404-9.

4. Zahar JR, Clec'h C, Tafflet M, Garrouste-Orgeas M, Jamali S, Mourvillier B, et al. Is methicillin resistance associated with a worse prognosis in Staphylococcus aureus ventilator-associated pneumonia? Clin Infect Dis 2005; 41: 1224-1231.

5. Dusemund F, Chronis J, Baty F, Albrich WC, Brutsche MH. The outcome of community-acquired pneumonia in patients with chronic lung disease: a case-control study. Swiss Med Wkly. 2014;144:w1401.

6. Mahoney FI, Barthel DW. Functional evaluation: the Barthel index. Md State Med J. 1965;14:61-5.

7. Hugh-Jones P, Lambert AV. A simple standard exercise test and its use for measuring exertion dyspnoea. Br Med J. 1952;1:65-71.

8. Yamana H, Moriwaki M, Horiguchi H, Kodan M, Fushimi K, Yasunaga H. Validity of diagnoses, procedures, and laboratory data in Japanese administrative data. J Epidemiol. 2017;27:476-82.

9. Shindo Y, Sato S, Maruyama E, Ohashi T, Ogawa M, Imaizumi K, et al. Comparison of severity scoring systems A-DROP and CURB-65 for communityacquired pneumonia. Respirology. 2008;13:731-5.

10. Metlay JP, Waterer GW, Long AC, Anzueto A, Brozek J, Crothers K, Cooley $L A$, et al. Diagnosis and treatment of adults with community-acquired pneumonia. An official clinical practice guideline of the American Thoracic Society and Infectious Diseases Society of America. Am J Respir Crit Care Med. 2019;200:e45-67.

11. Hennessy S, Bilker WB, Berlin JA, Strom BL. Factors influencing the optimal control-to-case ratio in matched case-control studies. Am J Epidemiol. 1999;149:195-7.

12. Knol MJ, Janssen KJ, Donders AR, Egberts AC, Heerdink ER, Grobbee $\mathrm{DE}$, et al. Unpredictable bias when using the missing indicator method or complete case analysis for missing confounder values: an empirical example. J Clin Epidemiol. 2010;63:728-36.
13. Groenwold RH, White IR, Donders AR, Carpenter JR, Altman DG, Moons KG. Missing covariate data in clinical research: when and when not to use the missing-indicator method for analysis. CMAJ. 2012;184:1265-9.

14. Jung WJ, Kang YA, Park MS, Park SC, Leem AY, Kim EY, et al. Prediction of methicillin-resistant Staphylococcus aureus in patients with non-nosocomial pneumonia. BMC Infect Dis. 2013;13:370.

15. Jwa H, Beom JW, Lee JH. Predictive factors of methicillin-resistant Staphylococcus aureus infection in elderly patients with community-onset pneumonia. Tuberc Respir Dis (Seoul). 2017;80:201-9.

16. Gross AE, Van Schooneveld TC, Olsen KM, Rupp ME, Bui TH, Forsung E, et al. Epidemiology and predictors of multidrug-resistant community acquired and health care-associated pneumonia. Antimicrob Agents Chemother. 2014;58:5262-8.

17. van Hal SJ, Jensen SO, Vaska VL, Espedido BA, Paterson DL, Gosbell IB. Predictors of mortality in Staphylococcus aureus bacteremia. Clin Microbiol Rev. 2012;25:362-86.

18. Inagaki K, Lucar J, Blackshear C, Hobbs CV. Methicillin-susceptible and methicillin-resistant Staphylococcus aureus bacteremia: nationwide estimates of 30-day readmission, in-hospital mortality, length of stay, and cost in the US. Clin Infect Dis. 2019;69:2112-8.

19. Cosgrove SE, Sakoulas G, Perencevich EN, Schwaber MJ, Karchmer AW, Carmeli Y. Comparison of mortality associated with methicillin-resistant and methicillin-susceptible Staphylococcus aureus bacteremia: a metaanalysis. Clin Infect Dis. 2003;36:53-9.

20. Ott E, Bange FC, Reichardt C, Graf K, Eckstein M, Schwab F, et al. Costs of nosocomial pneumonia caused by meticillin-resistant Staphylococcus aureus. J Hosp Infect. 2010;76:300-3.

21. Taneja C, Haque N, Oster G, Shorr AF, Zilber S, Kyan PO, et al. Clinical and economic outcomes in patients with community-acquired Staphylococcus aureus pneumonia. J Hosp Med. 2010;5:528-34.

22. Klein EY, Jiang W, Mojica N, Tseng KK, McNeill R, Cosgrove SE, et al. National costs associated with methicillin-susceptible and methicillinresistant Staphylococcus aureus hospitalizations in the United States, 2010-2014. Clin Infect Dis. 2019;68:22-8.

23. Fried LP, Tangen CM, Walston J, Newman AB, Hirsch C, Gottdiener J, et al. Frailty in older adults: evidence for a phenotype. J Gerontol A Biol Sci Med Sci. 2001;56:146-56.

24. Oda K, Yatera K, Fujino Y, Kido T, Hanaka T, Sennari K, et al. Respiratory comorbidities and risk of mortality in hospitalized patients with idiopathic pulmonary fibrosis. Respir Investig. 2018;56:64-71.

25. Taylor JK, Fleming GB, Singanayagam A, Hill AT, Chalmers JD. Risk factors for aspiration in community-acquired pneumonia: analysis of a hospitalized UK cohort. Am J Med. 2013;126:995-1001.

26. Neupane B, Walter SD, Krueger P, Marrie T, Loeb M. Predictors of inhospital mortality and re-hospitalization in older adults with community-acquired pneumonia: a prospective cohort study. BMC Geriatr. 2010;10:22.

\section{Publisher's Note}

Springer Nature remains neutral with regard to jurisdictional claims in published maps and institutional affiliations.

\footnotetext{
Ready to submit your research? Choose BMC and benefit from:

- fast, convenient online submission

- thorough peer review by experienced researchers in your field

- rapid publication on acceptance

- support for research data, including large and complex data types

- gold Open Access which fosters wider collaboration and increased citations

- maximum visibility for your research: over $100 \mathrm{M}$ website views per year
}

At BMC, research is always in progress.

Learn more biomedcentral.com/submissions 